\title{
ENDO AND ECTO PARASITE PREVALENCE AND ABUNDANCE IN SOME FISH SPECIES FROM AKOMOJE, OGUN RIVER SOUTH-WEST NIGERIA
}

\author{
Oghenochuko M.O'a,b*, Ezeri, G.N.O²b, Takeet M.I. ${ }^{3 c}$, Adeosun F.I. ${ }^{4 b}$, Disu I ${ }^{5 b}$ and Ogbia, \\ C.F $\mathbf{F}^{\mathbf{b}}$
}

\begin{abstract}
${ }^{a}$ Animal Science Programme, Department of Agriculture, Landmark University, PMB1001, Omu-Aran, Kwara State, NIGERIA. Email: oghenochuko.oghenebrorhie@1mu.edu.ng ${ }^{1}$

${ }^{b}$ College of Environmental Resources Management, Federal University of Agriculture, Abeokuta, PMB 2240, Ogun State, NIGERIA. Email: godfreyezeri@gmail.com²; adeosunfi@yahoo.com ${ }^{4}$; disu.ismail@yahoo.com ${ }^{5}$; thorlar26@gmail.com ${ }^{6}$

${ }^{c}$ College of Veterinary Medicine, Federal University of Agriculture, Abeokuta, PMB 2240, Ogun State, NIGERIA. Email: takeetmi@ funaab.edu.ng ${ }^{3}$

Corresponding author: oghenochuko.oghenebrorhie@1mu.edu.ng

Received: $3^{\text {rd }}$ May $2019 \quad$ Accepted: $17^{\text {th }}$ Aug 2020

DOI: https://doi.org/10.22452/mjs.vol39no3.1

Published: $31^{\text {st }}$ Oct 2020
\end{abstract}

\begin{abstract}
Parasites are the second most abundant microorganisms that infect and cause disease in wild and cultured fish after bacteria. The study investigated the parasite prevalence, abundance, mean intensity and dominance in some fresh water fish from Akomoje, Ogun River, Nigeria from February to May, 2016. Eight fish species were collected and identified to the species level. Experimental fish were measured and weighed. Endo- and ectoparasites were examined for; from Skin/scale, dorsal and caudal fins, gills, intestine and stomach of fish. Water sample was collected from shore, mid and extreme of the landing site and also analysed for parasite abundance. Prevalence of parasite in all fish species varied slightly with size. Myxozoan group revealed the highest dominance of ecto- and endo-parasites in virtually all fish species while mean intensity and abundance of Myxozoan spp. was highest in Oreochromis niloticus and Hemichromis fasciatus. Highest case of a single species of ectoand endo-parasite in a fish sample was that of Nematode larva in Chrysicthys nigrodigitatus (41.43\%) and Trichocerca sp. (Rotifera) in Mormyrus rume (52.9\%).Water analysis revealed three parasite groups that were present in the sampled fish. Conclusively, Akomoje landing site of Ogun River has a rich burden of parasites.
\end{abstract}

Keywords: Endoparasites, Ectoparasites, Ogun River, Dominance, Abundance, Oreochromis niloticus, Hemichromis fasciatus, Chrysicthys nigrodigitatus

\section{INTRODUCTION}

Fish is a very important source of nutrients of animal origin for varying healthy diets. It is a cheap source of animal protein and thus within reach of the average citizen of any nation (Mohanty, 2015). Fish demand is constantly on the increase and this is due among other reasons to the everincreasing human population, high cost of other sources of animal protein and issues of disease and infections associated with the consumption of other sources of animal protein (Tavarez-Dias \& Martins, 2017). The increasing population coupled with urbanization have resulted to problem of aquatic pollution and a corresponding prevalence of parasites and diseases in wild fish populations. Increasing aquatic environmental dynamics play a key role in determining where the hosts (fish or other aquatic organisms), parasites and other 
microbial pathogens exist (Zarlenga et al., 2014). According to Laffertty \& Kuris (2005), change in aquatic habitat has resulted to conditions suitable for the spread of trematodes.

Furthermore, fish serves as hosts for disease-causing parasites of man and some animals. Wild fish species have high probability of parasitic infestation and other microorganisms, but in most cases, they do not cause noticeable harm to the host. Few documented evidences exist on the pathogenicity and mortality-causing ability of parasites to the fish population, which could be due to the unnoticeable negative impacts of this parasites (Roberts, 2001). For instance, Neorickettsia helminthoeca the causative agent of salmon poisoning of dogs and human is harboured by the trematode called Nanophyetus salmincola, a parasite of fish. The dogs or human get infected by ingestion of metacercaria in infected fish. Parasites infestation in wild fishes are often recognise by fishermen or consumers only when they are so obvious as to cause reduction in the aesthetic value of the fish leading to rejection of fish (Roberts, 1995).

In Nigeria, extensive study has been carried out on parasites prevalence in fish (Okoye et al., 2014; Biu et al., 2014; Ejere et al., 2014; Uruku \& Adikwu, 2017; Ani et al., 2017; Abba et al., 2018) but only the study of Adeogun et al. (2014) is available in the study area. Aside the fact that very minimal study exist in the study area, none is available on the parasite prevalence in wild fish. Hence, the study was aimed to investigate and provide documented evidence on the prevalence of endo and ecto-parasites of some wild fish species in the Ogun State Nigeria.

\section{MATERIALS AND METHODS}

\subsection{Location}

Ogun River (Figure 1) was selected as the study location. The choice of Ogun River as the study site is because the study was a pilot study and nearness of location was a factor. Also, not much have been done on the parasite burden of this water body.River Ogun, situated in the Southwest of Nigeria, discharges into the Lagos Lagoon. The river rises in Oyo State near Shaki at coordinate's $8^{\circ} 41^{\prime} 0^{\prime \prime} \mathrm{N} 3^{\circ} 28^{\prime} 0^{\prime \prime} \mathrm{E} /$ $8.68333^{\circ} \mathrm{N} 3.46667^{\circ} \mathrm{E}$ and flows through Ogun State into Lagos State. The river is crossed by the Ikere Gorge Dam in the Iseyin local government area of Oyo State. The reservoir capacity is 690 million cubic metres $(560,000 \mathrm{acre} / \mathrm{ft})$. The reservoir abuts the Old Oyo National Park, providing recreational facilities for tourists, and the river flows through the park.

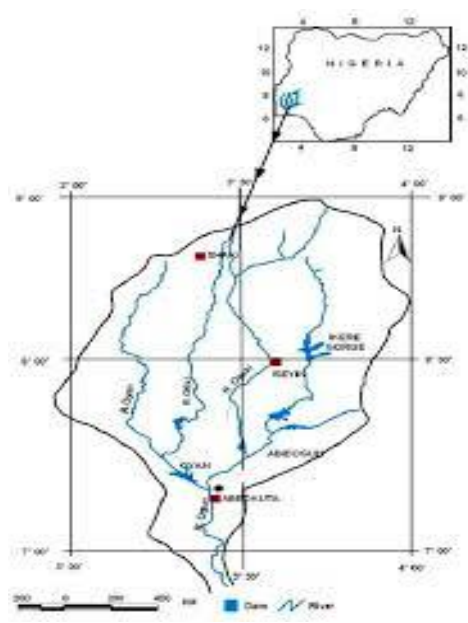

Figure 1. Map of River Ogun drainage basin (Source: Oke et al. (2012)) 


\subsection{Collection of Fish Sample}

A total of eight fish species (Mormyrus rume, Chrysichthys nigrodigitatus, Sarotherodon galilaeus, Brycinus macrolepidous, Hemichromis fasciatus, Tilapia mariae, Tilapia zilli and Oreochromis niloticus ) totalling 126 fish were purchased from local fishermen from landing sites at the Ogun River and transported in bucket filled with ice for preservation to the Parasitology laboratory of the Microbiology and Parasitology Department, College of Veterinary Medicine of the Federal University of Agriculture, Abeokuta. Further laboratory examination and analysis were then carried out.

\subsection{Identification of Fish Sample}

The fish samples were identified by experts in the Department of Aquaculture and Fisheries Management. The physical features of the fish which includes: the fins, body form, mouth type, colour etc. were used to identify the fish samples. Fish identification key by Idodo-Umeh (2003) was then used to confirm species identity.

\subsection{Laboratory Activities}

\subsubsection{Measurement of fish sample}

Morphometric characteristics of length (total and standard) and weight (Wt) of specimens were the major parameters used to categorise the fish samples into sizes. Fish length measurement were taken using measuring board to the nearest $0.1 \mathrm{~cm}$ Fish weights were measured by the use of a digital weighing balance (S. Mettler Electronic Compact Balance).

\subsubsection{Collection and examination of specimen}

Specimens were collected from the skin/scale, dorsal fin, gills and caudal fin. Skin/scale smear was collected aseptically by scrapping of the skin/scale using sterile scalpel blade, cutting the fins into separate sample bottles and refrigerated at $4^{\circ} \mathrm{C}$ till analysis. Skin/scales, caudal fin and dorsal fin of fish specimen were observed for ectoparasites using a light microscope.

The ventral side of the fish samples were aseptically dissected using sterile scalpel blade to expose the internal organs of the specimen. The alimentary canal of all fish samples were removed and cut into bits. The skin/scale smear, caudal and dorsal fin and contents of the gastrointestinal tract were washed into Petri-dish containing saline solution $(0.9 \mathrm{ml})$ to resuscitate the parasite. Stomachs and intestines were aseptically removed and dissected to reveal contents and washed into petri-dishes with normal saline. Examination of endo parasites was by the techniques of Bichi \& Dawaki (2010).

\subsubsection{Identification of parasite}

Parasites collected were identified using their distinctive body shapes and morphological features. Resuscitated parasites were grouped and identified using taxonomic guides by Paperna (1996), counted and recorded.

\subsection{Determination of Parasite Parameters}

The mean intensity, abundance and dominance of the ecto-parasites from the fish species and water samples were determined according to the method of Paperna (1980). 


\subsubsection{Mean intensity of Parasite}

$$
\text { Mean intensity }=\frac{\text { Total no. of collected parasites }}{\text { No. of infected fish samples }}
$$

\subsubsection{Abundance of Parasite}

$$
\text { Abundance }=\frac{\text { Total no. of collected parasites }}{\text { No. of host fish examined }}
$$

\subsubsection{Dominance of Parasite}

$$
\text { Dominance }=\frac{\mathrm{n}}{\text { Nsum }}
$$

Where:

$\mathrm{n}=$ abundance of a particular species,

$\mathrm{N}$ sum $=$ sum of the abundance of all parasite species found)

\subsubsection{Calculation of prevalence of Parasites}

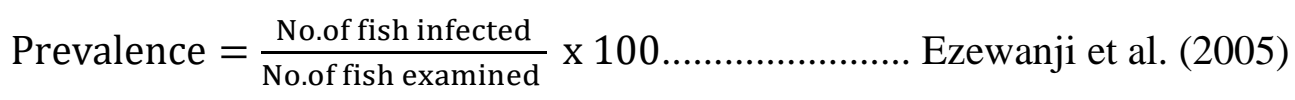

\subsection{Water Analysis}

Analyses of the water from three zones at the landing site were determined using sedimentation method. $10 \mathrm{ml}$ of the water sample was poured into a test tube and centrifuge with a centrifuge machine for 5 minute and pasteur pipette was used to take the suspended particles, placed on a clear slide and viewed under microscope X100 magnification.

\subsection{Statistical Analysis}

All data were presented as simple percentile incidence (\%). Mean intensity of infection was presented as percentage infected fish divided by 100. Data obtained were subjected to two way analysis of variance (ANOVA). Student T-test was used to determine the significant relationship between mean intensity and abundance using Statistical Package for Social Science software.

\section{RESULTS}

\subsection{Size Distribution and Prevalence of Parasite}

Size distribution and percentage of infected fish in the study are presented in Table 1. Chrysichthys nigrodigitatus had the highest number of fish examined and also the highest percentage of infected fish (36 fish sample were examined of which $15.08 \%$ were infected). This was followed by Brycinus macrolepidotus with a total of 18 fish examined and percentage infected $4.76 \%$ and the least of which was Mormyrus rume having a total of 9 fish examined with a percentage of $2.38 \%$ infected. 
Table 1. Size distribution and prevalence of parasite

\begin{tabular}{|c|c|c|c|c|c|c|}
\hline Fish species & $\begin{array}{c}\text { Length } \\
\text { class } \\
(\mathrm{cm})\end{array}$ & $\begin{array}{c}\text { Mean } \\
(\mathbf{L} \pm \text { SD })\end{array}$ & $\begin{array}{c}\text { Mean } \\
(\mathbf{W} \pm \text { SD })\end{array}$ & $\begin{array}{c}\text { No. } \\
\text { examined }\end{array}$ & $\begin{array}{c}\text { No. } \\
\text { Infected }\end{array}$ & $\begin{array}{c}\% \\
\text { infected }\end{array}$ \\
\hline \multirow[t]{3}{*}{ C. nigrodigitatus } & $\begin{array}{l}14- \\
16.9\end{array}$ & $15.56 \pm 0.81$ & $37.06 \pm 9.27$ & 24 & 12 & 50 \\
\hline & $\begin{array}{l}17- \\
17.9\end{array}$ & $18.73 \pm 0.64$ & $66.01 \pm 9.40$ & 10 & 5 & 50 \\
\hline & $\begin{array}{l}20- \\
22.9\end{array}$ & $21.0 \pm 0.71$ & $77.74 \pm 3.89$ & 2 & 2 & 100 \\
\hline \multirow[t]{3}{*}{ S. galilaeus } & 8- 13.9 & $12.33 \pm 1.45$ & $47.46 \pm 12.18$ & 8 & 4 & 50 \\
\hline & $\begin{array}{l}14- \\
19.9\end{array}$ & $17.04 \pm 1.70$ & $104.28 \pm 31.90$ & 7 & 3 & 42.9 \\
\hline & $\begin{array}{c}2- \\
25.9\end{array}$ & $21.75 \pm 2.47$ & $78.90 \pm 59.16$ & 2 & 1 & 50 \\
\hline \multirow[t]{3}{*}{ M. rume } & $\begin{array}{l}23- \\
28.9\end{array}$ & $24.24 \pm 1.48$ & $80.00 \pm 6.36$ & 2 & 1 & 50 \\
\hline & $\begin{array}{l}29- \\
32.9\end{array}$ & $30.83 \pm 1.86$ & $175.40 \pm 31.83$ & 4 & 0 & 0 \\
\hline & $\begin{array}{l}33- \\
36.9\end{array}$ & $33.40 \pm 0.36$ & $208.27 \pm 14.84$ & 3 & 2 & 66.7 \\
\hline \multirow[t]{3}{*}{ B.macrolepidotus } & $\begin{array}{l}14- \\
17.9\end{array}$ & $15.50 \pm 0.10$ & $30.98 \pm 8.65$ & 7 & 2 & 28.6 \\
\hline & $\begin{array}{l}18- \\
21.9\end{array}$ & $19.30 \pm 0.68$ & $76.78 \pm 15.38$ & 6 & 2 & 33.3 \\
\hline & $\begin{array}{l}22- \\
25.9\end{array}$ & $22.64 \pm 0.25$ & $132.15 \pm 16.86$ & 5 & 2 & 40 \\
\hline
\end{tabular}




$\begin{array}{lllllll}\text { H.fasciatus } & 11- & 13.08 \pm 1.55 & 51.21 \pm 13.74 & 6 & 5 & 83.3 \\ & 15.9 & & & & & \\ & 16- & 18.84 \pm 1.16 & 78.61 \pm 9.11 & 5 & 2 & 40 \\ 20.9 & & & & & \\ & 21- & 22.00 \pm 0.00 & 115.05 \pm 0.00 & 1 & 1 & 100 \\ 25.9 & & & & & \end{array}$

\begin{tabular}{|c|c|c|c|c|c|c|}
\hline \multirow[t]{2}{*}{ T. mariae } & $\begin{array}{l}10- \\
14.9\end{array}$ & $11.95 \pm 1.58$ & $36.99 \pm 18.56$ & 13 & 5 & 38.5 \\
\hline & $\begin{array}{l}20- \\
24.9\end{array}$ & $22.65 \pm 0.21$ & $197.05 \pm 14.64$ & 2 & 1 & 50 \\
\hline \multirow[t]{2}{*}{ O. niloticus } & $\begin{array}{l}12- \\
14.9\end{array}$ & $13.38 \pm 0.63$ & $52.63 \pm 12.21$ & 4 & 2 & 50 \\
\hline & $\begin{array}{l}15- \\
18.9\end{array}$ & $16.57 \pm 1.66$ & $82.07 \pm 35.24$ & 3 & 1 & 33.3 \\
\hline \multirow[t]{2}{*}{ T. zilli } & $\begin{array}{l}11- \\
13.9\end{array}$ & $12.21 \pm 0.88$ & $36.04 \pm 9.18$ & 7 & 4 & 57.1 \\
\hline & $\begin{array}{l}14- \\
16.9\end{array}$ & $14.68 \pm 0.51$ & $59.74 \pm 7.94$ & 5 & 5 & 100 \\
\hline
\end{tabular}

TOTAL

\subsection{Parasite Load of Fish Species Examined}

Result revealed 7 parasite groups comprising of 13 parasite species from fish species sampled. Dominance was highest in Trichocerca $\mathrm{sp}$ and Nematode larva (52.94 and $41.43 \%$ ) respectively. Least dominance of ecto-parasite was however recorded in Microcystis sp, Oedogonium sp, Pediastrum sp and Didinium with (1.43\%) and for endo-parasite in Ichthyophthimius sp, Pandorina sp and Polyaritha sp respectively $(0.73 \%)$ (Table 2$)$. Least dominance of endoparasites was recorded in Tilapia species. 
Table 2. Species composition, Mean intensity, Abundance and Dominance of Parasite and infected parts of fish.

\begin{tabular}{|c|c|c|c|c|c|c|}
\hline Fish species & Parasite group & Parasite species & $\begin{array}{c}\text { Infected } \\
\text { Part } \\
\end{array}$ & $\mathrm{MI} \pm \mathrm{SD}$ & $\mathrm{MA} \pm \mathrm{SD}$ & Dominance $(\%)$ \\
\hline \multirow[t]{6}{*}{ Chrysichthysnigrodigitatus } & \multirow[t]{2}{*}{ MYXOZOANS } & $\begin{array}{c}\text { Microcystis } \mathrm{sp}, \\
\text { Coelosphaerium } \mathrm{sp}, \\
\text { Closterium } \mathrm{sp}, \text { Oscillatoria } \\
\mathrm{sp}, \text { Polycystis } \mathrm{sp}, \\
\text { Oedogonium } \mathrm{sp} \\
\text { Pediastrum } \mathrm{sp}, \text { Zygenma } \\
\text { sp, Tetraspora } \mathrm{sp}, \\
\text { Mougeotia } \mathrm{sp},\end{array}$ & $\begin{array}{l}\text { Skin, } \\
\text { caudal fin }\end{array}$ & $1.74 \pm 0.10^{\mathrm{a}}$ & $0.92 \pm 0.32^{b}$ & $\begin{array}{c}1.43,8.57,4.28, \\
14.29,5.71,1.43, \\
1.43,4.28,2.86, \\
2.86\end{array}$ \\
\hline & & $\begin{array}{c}\text { Spirulina } \mathrm{sp}, \\
\text { Coelosphaerium } \mathrm{sp}, \\
\text { Oedogonium } \mathrm{sp}, \text { Polycystis } \\
\text { sp, Pleurotaenitium } \mathrm{sp}, \\
\text { Closterium } \mathrm{sp}, \text { Oscillatoria } \\
\text { sp, Tetraspedia } \mathrm{sp}\end{array}$ & $\begin{array}{l}\text { Intestine, } \\
\text { Gill }\end{array}$ & $2.47 \pm 0.83^{\mathrm{a}}$ & $1.31 \pm 1.21^{\mathrm{b}}$ & $\begin{array}{c}0.74,5.15,2.21 \\
15.44,2.94 \\
4.41,0.74,2.94\end{array}$ \\
\hline & \multirow[t]{2}{*}{ NEMATODA } & Nematode egg, Colpoda sp & $\begin{array}{c}\text { Skin, } \\
\text { caudal fin }\end{array}$ & $1.89 \pm 0.10^{\mathrm{a}}$ & $1.00 \pm 0.21^{b}$ & $41.43,10$ \\
\hline & & Nematode egg & $\begin{array}{c}\text { Intestine, } \\
\text { stomach }\end{array}$ & $0.37 \pm 0.08^{\mathrm{a}}$ & $0.20 \pm 0.10^{\mathrm{a}}$ & 5.15 \\
\hline & \multirow[t]{2}{*}{ PROTOZOAN } & Didinium sp & $\begin{array}{l}\text { Skin, } \\
\text { caudal fin }\end{array}$ & $0.05 \pm 0.07^{\mathrm{a}}$ & $0.03 \pm 0.01^{\mathrm{a}}$ & 1.43 \\
\hline & & $\begin{array}{c}\text { Urostyla sp, Synura sp, } \\
\text { Frontonia } \mathrm{sp}\end{array}$ & $\begin{array}{l}\text { Intestine, } \\
\text { Gill }\end{array}$ & $0.26 \pm 0.05^{\mathrm{a}}$ & $0.14 \pm 0.09^{\mathrm{a}}$ & $0.74,1.47,0.74$ \\
\hline
\end{tabular}




\begin{tabular}{|c|c|c|c|c|c|c|}
\hline & ROTIFER & $\begin{array}{c}\text { Polyarthra sp, Trichocerca } \\
\text { sp }\end{array}$ & $\begin{array}{l}\text { Intestine, } \\
\text { Gill }\end{array}$ & $2.95 \pm 0.84^{\mathrm{a}}$ & $1.56 \pm 2.18^{b}$ & $0.74,0.74$ \\
\hline & CRUSTACEAN & Eubranchipus sp & $\begin{array}{l}\text { Intestine, } \\
\text { Gill }\end{array}$ & $0.89 \pm 0.13^{\mathrm{a}}$ & $0.47 \pm 0.39^{b}$ & 12.5 \\
\hline & TREMATODA & Trematode eggs & $\begin{array}{l}\text { Intestine, } \\
\text { Gill }\end{array}$ & $0.05 \pm 0.02^{\mathrm{a}}$ & $0.03 \pm 0.03^{\mathrm{a}}$ & 0.74 \\
\hline & CESTODA & Cestode egg & $\begin{array}{l}\text { Intestine, } \\
\text { Gill }\end{array}$ & $0.16 \pm 0.04^{\mathrm{a}}$ & $0.08 \pm 0.06^{\mathrm{b}}$ & 2.21 \\
\hline \multirow[t]{6}{*}{ Sarotherodongalilaeus } & MYXOZOANS & $\begin{array}{c}\text { Microcystis sp, Rivularian } \\
\text { sp, Coelosphaerium } \mathrm{sp} \\
\text { Polycystis } \mathrm{sp}\end{array}$ & $\begin{array}{l}\text { Scale, } \\
\text { dorsal fins }\end{array}$ & $1.00 \pm 0.03^{\mathrm{a}}$ & $0.41 \pm 0.10^{\mathrm{a}}$ & $\begin{array}{l}9.09,9.09 \\
9.09,18.18\end{array}$ \\
\hline & & $\begin{array}{l}\text { Closteriumsp, Zygenma sp, } \\
\text { Polycystis sp, Tetraspedia } \\
\text { sp, Merismopedia sp }\end{array}$ & $\begin{array}{l}\text { Stomach, } \\
\text { intestine, } \\
\quad \text { gills }\end{array}$ & $2.20 \pm 0.17^{\mathrm{a}}$ & $0.65 \pm 0.30^{\mathrm{b}}$ & $\begin{array}{c}20.69,3.45 \\
6.89,3.45,3.45\end{array}$ \\
\hline & TREMATODA & Trematodes egg & $\begin{array}{c}\text { Scale, } \\
\text { dorsal fins }\end{array}$ & $0.14 \pm 0.05^{\mathrm{a}}$ & $0.06 \pm 0.12^{\mathrm{a}}$ & 9.09 \\
\hline & PROTOZOAN & $\begin{array}{l}\text { Difflugia sp, Uroglena sp, } \\
\text { Spirostomum } \mathrm{sp}\end{array}$ & $\begin{array}{l}\text { Scale, } \\
\text { dorsal fins }\end{array}$ & $0.71 \pm 0.51^{\mathrm{a}}$ & $0.41 \pm 0.10^{\mathrm{a}}$ & $\begin{array}{l}9.09,18.18 \\
18.18\end{array}$ \\
\hline & & $\begin{array}{l}\text { Volvox } \mathrm{sp}, \text { Didinium sp, } \\
\text { Euglena sp, Frontonia sp }\end{array}$ & $\begin{array}{l}\text { Stomach, } \\
\text { intestine }\end{array}$ & $3.40 \pm 1.12^{\mathrm{a}}$ & $1.00 \pm 0.28^{b}$ & $\begin{array}{c}48.27,3.45 \\
3.45,3.45\end{array}$ \\
\hline & NEMATODA & Nematode eggs & $\begin{array}{l}\text { Stomach, } \\
\text { intestine }\end{array}$ & $0.20 \pm 0.09^{\mathrm{a}}$ & $0.06 \pm 0.04^{\mathrm{a}}$ & 3.45 \\
\hline \multirow[t]{2}{*}{ Mormyrusrume } & MYXOZOANS & Selenastrum $\mathrm{sp}$ & $\begin{array}{l}\text { Skin, } \\
\text { caudal } \\
\text { fins }\end{array}$ & $0.03 \pm 0.12^{\mathrm{a}}$ & $0.11 \pm 0.04^{\mathrm{a}}$ & 33.33 \\
\hline & & $\begin{array}{l}\text { Coelosphaerium } \mathrm{sp} \\
\text { Spirulina } \mathrm{sp}\end{array}$ & $\begin{array}{l}\text { Stomach, } \\
\text { intestine }\end{array}$ & $0.50 \pm 0.10^{\mathrm{a}}$ & $0.22 \pm 0.15^{\mathrm{a}}$ & $2.94,2.94$ \\
\hline
\end{tabular}




\begin{tabular}{|c|c|c|c|c|c|c|}
\hline & PROTOZOAN & $\begin{array}{l}\text { Uroglena sp, } \\
\text { Ichthophthrinus sp } \\
\text { Volvox sp }\end{array}$ & $\begin{array}{c}\text { Skin, } \\
\text { caudal } \\
\text { fins } \\
\text { Stomach, } \\
\text { intestine, } \\
\text { gills }\end{array}$ & $2.00 \pm 0.23^{\mathrm{a}}$ & $0.88 \pm 0.57^{\mathrm{a}}$ & $33.33,33.34$ \\
\hline & CRUSTACEAN & Cyclops sp, Eubranchipus & $\begin{array}{l}\text { Stomach, } \\
\text { intestine }\end{array}$ & $1.50 \pm 0.32^{\mathrm{a}}$ & $0.66 \pm 0.18^{a}$ & $11.76,5.89$ \\
\hline & ROTIFERS & Trichocerca sp & $\begin{array}{l}\text { Stomach, } \\
\text { intestine, } \\
\text { gills }\end{array}$ & $4.50 \pm 1.84^{\mathrm{a}}$ & $2.00 \pm 0.98^{b}$ & 52.94 \\
\hline \multirow[t]{3}{*}{ Brycinusmacrolepidotus } & MYXOZOANS & $\begin{array}{c}\text { Polycystis sp, Anabaena sp, } \\
\text { Tetraspora } \mathrm{sp}\end{array}$ & $\begin{array}{l}\text { Scale, } \\
\text { dorsal fins }\end{array}$ & $1.40 \pm 0.17^{\mathrm{a}}$ & $0.39 \pm 0.15^{b}$ & $\begin{array}{l}28.57,28.57 \\
42.86\end{array}$ \\
\hline & & $\begin{array}{c}\text { Ulothrix sp, Aphanocapsa } \\
\text { sp, Cosmarium sp, } \\
\text { Pediastrum sp, Spirotaenia } \\
\text { sp, Coelosphaerium sp, } \\
\text { Epithemia sp, Oedogonium } \\
\text { sp }\end{array}$ & $\begin{array}{l}\text { Stomach, } \\
\text { intestine }\end{array}$ & $2.89 \pm 1.42^{\mathrm{a}}$ & $1.44 \pm 0.72^{\mathrm{a}}$ & $\begin{array}{c}4.26,6.38,2.13, \\
2.13,10.64 \\
12.77,6.38 \\
10.64\end{array}$ \\
\hline & PROTOZOAN & $\begin{array}{c}\text { Volvox sp, Carchesium } \mathrm{sp} \text {, } \\
\text { Dictyostelium } \mathrm{sp}\end{array}$ & $\begin{array}{l}\text { Stomach, } \\
\text { intestine }\end{array}$ & $2.33 \pm 1.18^{\mathrm{a}}$ & $11.67 \pm 1.34^{\mathrm{b}}$ & $4.26,2.13,38.28$ \\
\hline \multirow[t]{2}{*}{ Hemichromis fasciatus } & MYXOZOANS & $\begin{array}{c}\text { Oscillatoria } \mathrm{sp}, \\
\text { Microspora } \mathrm{sp}, \\
\text { Merismopedia } \mathrm{sp}\end{array}$ & $\begin{array}{l}\text { Scale, } \\
\text { dorsal fin, } \\
\text { caudal fin }\end{array}$ & $1.43 \pm 0.17^{\mathrm{a}}$ & $0.83 \pm 0.08^{a}$ & $45.45,36.37,9.09$ \\
\hline & & $\begin{array}{c}\text { Tetraspedia sp, } \\
\text { Coelosphaerium } \mathrm{sp}, \\
\text { Pleurotaenitium } \mathrm{sp},\end{array}$ & $\begin{array}{l}\text { Stomach, } \\
\text { intestine }\end{array}$ & $10.22 \pm 1.89^{\mathrm{a}}$ & $7.67 \pm 1.74^{\mathrm{a}}$ & $\begin{array}{c}40.37,3.67 \\
3.67,10.09 \\
26.61\end{array}$ \\
\hline
\end{tabular}


Oedogonium sp, Polycystis

$\mathrm{sp}$

\begin{tabular}{|c|c|c|c|c|c|c|}
\hline & PROTOZOAN & Laxodes sp & $\begin{array}{l}\text { Skin, } \\
\text { caudal fin }\end{array}$ & $0.29 \pm 0.14^{\mathrm{a}}$ & $0.17 \pm 0.12^{b}$ & 9.09 \\
\hline & NEMATODA & Nematode larvae & $\begin{array}{l}\text { Stomach, } \\
\text { intestine }\end{array}$ & $1.78 \pm 0.32^{\mathrm{a}}$ & $1.33 \pm 0.18^{\mathrm{a}}$ & 14.67 \\
\hline & TREMATODA & Trematode eggs & $\begin{array}{l}\text { Stomach, } \\
\text { intestine }\end{array}$ & $0.11 \pm 0.10^{\mathrm{a}}$ & $0.08 \pm 0.05^{\mathrm{a}}$ & 0.92 \\
\hline \multirow[t]{5}{*}{ Tilapia mariae } & MYXOZOANS & $\begin{array}{c}\text { Tetraspora } \mathrm{sp} \\
\text { Coelosphaerium } \mathrm{sp}\end{array}$ & $\begin{array}{l}\text { Skin, } \\
\text { caudal fin }\end{array}$ & $1.2 \pm 0.20^{\mathrm{a}}$ & $0.47 \pm 0.14^{b}$ & $66.67,11.11$ \\
\hline & & $\begin{array}{l}\text { Closterium sp, Cosmarium } \\
\text { sp, Polycystissp, Zygenma } \\
\text { sp, Tetraspedia sp, } \\
\text { Coelosphaerium sp }\end{array}$ & $\begin{array}{l}\text { Stomach, } \\
\text { intestine, } \\
\text { gills }\end{array}$ & $9.50 \pm 2.84^{\mathrm{a}}$ & $6.33 \pm 1.26^{b}$ & $\begin{array}{l}35.04,2.19 \\
2.92,1.46 \\
24.81,2.92\end{array}$ \\
\hline & NEMATODA & $\begin{array}{c}\text { Tribonema sp, Nematode } \\
\text { larvae }\end{array}$ & $\begin{array}{l}\text { Skin, } \\
\text { dorsal fin }\end{array}$ & $1.70 \pm 0.31^{\mathrm{a}}$ & $0.67 \pm 0.07^{b}$ & $11.11,11.11$ \\
\hline & PROTOZOAN & $\begin{array}{l}\text { Pandorina } \mathrm{sp}, \text { Volvox } \mathrm{sp} \\
\text { Ichthyophthimius sp }\end{array}$ & $\begin{array}{l}\text { Stomach, } \\
\text { intestine, } \\
\text { gill }\end{array}$ & $0.80 \pm 1.14^{\mathrm{a}}$ & $0.53 \pm 0.24^{\mathrm{a}}$ & $0.73,4.38,0.73$ \\
\hline & ROTIFERS & $\begin{array}{c}\text { Trichocerca sp, Polyaritha } \\
\text { sp }\end{array}$ & $\begin{array}{l}\text { Stomach, } \\
\text { intestine }\end{array}$ & $3.40 \pm 1.86^{\mathrm{a}}$ & $2.27 \pm 0.10^{\mathrm{a}}$ & $24.09,0.73$ \\
\hline \multirow[t]{2}{*}{ Orechromisniloticus } & MYXOZOANS & $\begin{array}{c}\text { Tetraspora } \mathrm{sp} \\
\text { Coelosphaerium } \mathrm{sp}\end{array}$ & $\begin{array}{l}\text { Skin, } \\
\text { dorsal fin }\end{array}$ & $2.33 \pm 0.89^{\mathrm{a}}$ & $1.00 \pm 0.09^{b}$ & $85.71,14.29$ \\
\hline & & $\begin{array}{l}\text { Closterium sp, Tetraspedia } \\
\text { sp, Aphanocapsa sp, } \\
\text { Oedogonium sp, Polycystis } \\
\text { sp, }\end{array}$ & $\begin{array}{l}\text { Stomach, } \\
\text { intestine }\end{array}$ & $4.44 \pm 3.10^{\mathrm{a}}$ & $5.71 \pm 3.01^{b}$ & $\begin{array}{c}5,31.66,11.66 \\
16.67,1.56\end{array}$ \\
\hline
\end{tabular}




\begin{tabular}{|c|c|c|c|c|c|c|}
\hline & PROTOZOAN & $\begin{array}{c}\text { Eudorina } \mathrm{sp}, \text { Dictyostelium } \\
\mathrm{sp} \text {, Volvox } \mathrm{sp}\end{array}$ & $\begin{array}{l}\text { Stomach, } \\
\text { intestine }\end{array}$ & $2.11 \pm 0.76^{\mathrm{a}}$ & $2.71 \pm 0.13^{\mathrm{a}}$ & $\begin{array}{c}11.66,6.66, \\
13.33\end{array}$ \\
\hline & NEMATODA & Nematode egg & $\begin{array}{l}\text { Stomach, } \\
\text { intestine }\end{array}$ & $0.11 \pm 1.48^{\mathrm{a}}$ & $0.14 \pm 0.21^{\mathrm{a}}$ & 3.33 \\
\hline \multirow[t]{5}{*}{ Tilapia zilli } & MYXOZOANS & $\begin{array}{l}\text { Closterium sp, Polycystis } \\
\text { sp, Tetraspedia sp, } \\
\text { Ulothrix sp, Pediastrium sp }\end{array}$ & $\begin{array}{l}\text { Skin, } \\
\text { dorsal fin, } \\
\text { caudal fin }\end{array}$ & $1.67 \pm 0.57^{\mathrm{a}}$ & $1.25 \pm 0.34^{\mathrm{a}}$ & $\begin{array}{c}52.94,17.65 \\
5.88,5.88,5.88\end{array}$ \\
\hline & & $\begin{array}{l}\text { Closterium sp, Anabaena } \\
\text { sp, Ulothrix sp, } \\
\text { Oscillatoria } \mathrm{sp}, \text { Epithemia } \\
\text { sp, Microspora } \mathrm{sp} \\
\text { Oedogonium } \mathrm{sp}, \\
\text { Tetraspedia } \mathrm{sp}\end{array}$ & $\begin{array}{l}\text { Stomach, } \\
\text { intestine, } \\
\text { gills }\end{array}$ & $4.44 \pm 1.48^{\mathrm{a}}$ & $3.33 \pm 1.39^{\mathrm{a}}$ & $\begin{array}{c}18.18,2.27, \\
6.82,2.27, \\
11.36,22.73,25, \\
2.27\end{array}$ \\
\hline & \multirow[t]{2}{*}{ PROTOZOAN } & $\begin{array}{c}\text { Pleodrina } \mathrm{sp}, \text { Dileptus } \mathrm{sp} \\
\text { Endorina } \mathrm{sp}\end{array}$ & $\begin{array}{l}\text { Skin, } \\
\text { caudal fin }\end{array}$ & $0.33 \pm 0.18^{\mathrm{a}}$ & $0.25 \pm 0.11^{\mathrm{a}}$ & $5.88,5.88,5,88$ \\
\hline & & Pandorina sp, Frontonia sp & $\begin{array}{l}\text { Stomach, } \\
\text { intestine }\end{array}$ & $0.33 \pm 0.18^{\mathrm{a}}$ & $0.25 \pm 0.72^{\mathrm{a}}$ & $2.27,4.56$ \\
\hline & ROTIFERS & Testudinella $\mathrm{sp}$ & $\begin{array}{l}\text { Stomach, } \\
\text { intestine, } \\
\text { gills }\end{array}$ & $0.11 \pm 0.12^{\mathrm{a}}$ & $0.08 \pm 0.15^{\mathrm{a}}$ & 2.27 \\
\hline
\end{tabular}




\subsection{Distribution of Parasites in Water Body}

The result revealed that Myxozoan spp. were found to be predominant. Figure 2 showed that Myxozoan spp. represented $76.00 \%$ of all the parasites while Crustaceans were least represented with only one (1) parasite species (Table 3). Protozoans recorded about $27.00 \%$ of the parasite groups with six (6) parasite species.

Table 3. Location distribution of Parasites in water from Akomoje, Ogun River

\begin{tabular}{|c|c|c|c|c|c|}
\hline \multirow{2}{*}{ Parasite Group } & \multirow{2}{*}{ Parasite species } & \multicolumn{3}{|c|}{ ZONE } & \multirow{2}{*}{ Total } \\
\hline & & Shore & Middle & Extreme & \\
\hline \multirow[t]{11}{*}{ MYXOZOANS } & Oscillatoria $\mathrm{sp}$ & 1 & 1 & 0 & 2 \\
\hline & Spirulina sp & 38 & 16 & 11 & 65 \\
\hline & Merismopedia $\mathrm{sp}$ & 4 & 10 & 17 & 31 \\
\hline & Oedogonium sp & 22 & 34 & 60 & 116 \\
\hline & Closterium sp & 3 & 0 & 0 & 3 \\
\hline & Ankistrodesmus sp & 0 & 1 & 0 & 1 \\
\hline & Ulothrix sp & 0 & 0 & 1 & 1 \\
\hline & Zygenma sp & 0 & 2 & 0 & 2 \\
\hline & Docidium sp & 0 & 0 & 1 & 1 \\
\hline & Netrium $\mathrm{sp}$ & 0 & 3 & 0 & 3 \\
\hline & Pleurotaenitium sp & 1 & 2 & 0 & 3 \\
\hline \multirow[t]{6}{*}{ PROTOZOAN } & Pandorina sp & 4 & 0 & 38 & 42 \\
\hline & Bodo sp & 10 & 0 & 21 & 31 \\
\hline & Volvox sp & 0 & 1 & 0 & 1 \\
\hline & Clupods sp & 0 & 0 & 1 & 1 \\
\hline & Hartiminella sp & 0 & 3 & 0 & 3 \\
\hline & Didinium sp & 0 & 0 & 1 & 1 \\
\hline CRUSTACEAN & Cypridops sp & 0 & 0 & 1 & 1 \\
\hline TOTAL & & 83 & 73 & 152 & 308 \\
\hline
\end{tabular}




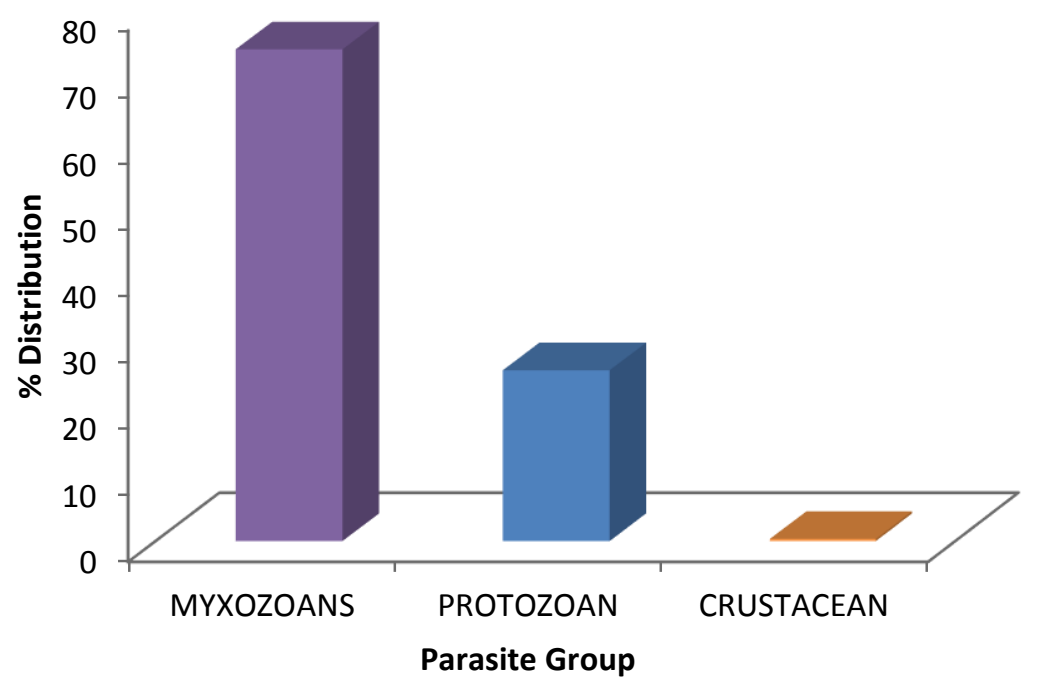

Figure 2. Percentage distribution of parasite within group in water from the study area.

\section{DISCUSSION}

Clear understanding of the spread and prevalence of parasites of wild fish is a recipe for formulation of effective control or elimination strategy for improving the health standard of human and profitable fish farming in Nigeria, hence this study has attempted to shed more light on the distribution and prevalence of wild fish and the parasites infecting them in Ogun State Nigeria.

The result of the study revealed the rich parasite burden of endo- and ectoparasites in the study area. Seven parasite groups consisting of 13 species were retrieved from eight fish species analysed, is a clear indication of high parasite diversity for a landing site. Omeji et al. (2010), also reported similar result in Heterobranchus longifilis obtained from the wild in Benue State. High prevalence of parasites in all fish species and sizes recorded in this study could be as a result of several factors prevailing such as but not limited to host fish sex, location of removal, age and size (Bichi \& Bizi, 2002) and the aquatic ecosystem (Lafferty \& Kuris, 2005). It may also be as a result of increased contact between the host and parasite.

However, significant variation was observed in parasite prevalence between various sizes of the different fish species with highest prevalence in bigger fish than in the smaller fish which revealed as high as $100 \%$ presence. Reasons could be as result of their foraging habit and the abundance food available and consumed by them. Goselle et al. (2008) reported a similar result for Clarias gariepinus and Tilapia zilli obtained from Lamingo Dam, Jos, Nigeria. Bichi \& Ibrahim (2009) however reported higher prevalence in the level of both external parasites and that found in the internal organs of the Tilapia zilli of smaller sizes in their survey of Tiga Lake, Kano, Nigeria. Reason for this difference could be attributed to the varying distribution of parasites in the different habitat which could be due to host-parasite interaction and the water quality parameters of dissolved oxygen, temperature and $\mathrm{pH}$ of the fish environment (Anderson, 1992). 
The highest percentage intensity of the Myxozoan spp. and its abundance in O.niloticus and $H$. fasciatus reported in this study is corroborated by the study of Tossavi et al. (2014), Ugbor et al. (2014). Also, Karvonen \& Valtonen (2004) and Okoye et al. (2014) documented the richness of the tropical fresh waters in their parasitic species burdens. Indication from this result is that such parasite burden in an ecosystem may pose high risk of infection to both fish and man who might feed on the fish species which serve as secondary host of human pathogenic parasites or where fish is a transport host of zoonotic parasites. However, the studies of Ekanem et al. (2011) and Ejere et al. (2014) are not in agreement with our findings.

Generally, there will be need to characterized these parasites molecularly in future to shed more light on their genetic diversity in the study area.

\section{CONCLUSION}

Akomoje landing site of Ogun River has a rich burden of ecto- and endoparasites mainly myxozoan, protozoan and crustacean.and thus we recommend that constant surveillance of the water body be carried out to know the prevalence of parasites in the water body in order to prevent possible food-borne parasitic disease outbreak. In addition, detailed study of the seasonal variations of parasites load in this water body is also recommended.

\section{ACKNOWLEGDEMENT}

The authors appreciates the laboratory technologist of the Department of Veterinary Microbiology and Parasitology of the College of Veterinary Medicine, Federal University of Agriculture, Abeokuta, Nigeria.

\section{REFERENCES}

Abba, A. M., Emere, M. C., Appah J., \& Omenesa, R. L. (2018). Helminth Parasites of Two Freshwater Fishes (Oreochromis niloticus and Clariasgariepinus) in Jibia Earth Dam, Katsina State, Nigeria. Global Journal of Science Frontier Reserch, 18(1): 35-41. Online ISSN: 2249-4626 \& Print ISSN: 0975-5896.

Adeogun, O.A., Oladosu, G.A., Akinwale, M.M.A., Okunade, O.A., Akintayo, I.A., Idika, N., Adeiga, A.A., Ezeugwu, S.M.C., Afocha, E.E., Peters, O.S., \& Odusanya, A.F. (2014). Identification, Distribution and Prevalence of Ecto-parasites Associated with Cultured Fish in Ogun State, Nigeria. Journal of Fisheries and Aquatic Science 9(5): 413-418.

DOI: $10.3923 /$ jas.2014.413.418.

Anderson, R.C. (1992). Nematodia parasites of verterbrates, their development and transmission. 650 p. Willingford: C.A.B international.

Ani, O.C., Nnamonu, E.I. \& Ejiogu, C. (2017). Prevalence of Intestinal Parasites of Fish Farmed and Harvested in Abakiliki, Nigeria: A Pointer to the Level of their Vulnerability. International Journal of Research in Pharmacy and Biosciences, 4(9): 7-10. ISSN 2394 - 5885 (Print) \& ISSN 2394-5893 (Online).

Bichi, A. H. \& Ibrahim, A.A. (2009). A survey of ecto and intestinal parasites of tilapia zilli (Gervias) in Tiga Lake, Kano, Northern Nigeria. Bayero Journal of Pure and Applied Sciences, 2(1): 79-82. 
Bichi, A.H. \& Bizi, A.G. (2002): Survey of ecto and endo parasites of fishes of Challawa George Dam. NISEB Journal, 2(3): 219-222.

Bichi, A.H. \& Dawaki, S.S. (2010). A survey of ecto parasites on the gills skin and fins of Oreochromis niloticus at Bagauda fish farm, Kano Nigeria.Bayero J. Pure Appl Sci., 3(1): 83- 86.

Biu, A.A., Diyaware, M.Y., Yakaka, W. \& Joseph, E. (2014). Survey of Parasites Infesting the Nile Tilapia (Oreochromis niloticus Linnaeus, 1758) from Lake Alau, Maiduguri, Nigeria. Nigerian Journal of Fisheries and Aquaculture, 2(2): 6 12. ISSN 2350-1537.

Ejere, V. C., Aguzie, O. I., Ivoke, N., Eke, F. N., Ezenwaji, N. E., Onoja, U. S., Eyo, J. E. \& Onoja, U. S. (2014). Parasitofauna of five freshwater fishes in a Nigerian freshwater ecosystem. Croatian Journal of Fisheries, 72(1): 17 - 24. DOI: 10.14798/72.1.682.

Ekanem, A.P., Eyo, V.O. \& Sampson, A.F. (2011). Parasite of landed fish from great Kwa River, Calaber, Cross river State, Nigeria. International Journal of Fisheries and Aquaculture, 3(12): 225-230. DOI: 105897/IJFA11.072. ISSN 200698.

Ezenwaji, N.E., Aguigwo, J.N.I., Philip, C.O. \& Ezenwaji, H.M.G. (2005). Helminthendo - parasites of mochokids in a tropical rainforest river system Animal Research International, 2(2): $\quad 346-352$.

Goselle, O.N., Shir, G.I., Udeh, E.O.,Abelau, M. \& Imandeh, G.N. (2008). Helminth parasites of Clariasgariepinus and Tilapia zilli at Lamingo dam, Jos, Nigeria. Science world Journal, 3(4): 23-28. ISSN 1597-6343.

Idodo-Umeh, G. (2003). Freshwater Fishes of Nigeria (Taxonomy, Ecological notes, Diet and Utilization). 232p. Benin City, Edo State: Idodo-Umeh publishers Limited.

Karvonen, A. \& Valtonen, E.T. (2004). Helminth assemblages of Whitefish (Coregonuslavaretus) in interconnected Lakes: Similarity as a function of species specific parasites and geographical separation. Journal of Parasitology, 90(3), 471-476.

Lafferty, K.D. \& Kuris, A.M. (2005). Parasitism and Environmental disturbances. In: Parasitism and Ecosystems. Thomas F., Renaud, F and Guegan, Jean-Francois (Eds.). 113 - 123pp. UK: Oxford University Press. Retrieved 17 Mar., 2019, from http://www.oxfordscholarship.com/ view/10.1093/acprof.oso/97801985 29873.001.0001/acprof9780198529873-chapter-8.

Mohanty, B. P. (2015). Nutritional Value of fish. In: Conspectus of Inland Fisheries Management. A.K. Das and D. Panda (Eds.).ICAR- Central Inland Fisheries Research Institute (Indian Council of Agricultural Research), Barrackpore, Kolkata, 700-120, W.B. ICAR.15-21pp.

Oke, M.O., Martins, O. \& Idowu, O. A. (2012). Monitoring of Groundwater Recharge for Flood Management. Hydrology for Disaster Management Special Publication of the Nigerian Association of Hydrological Sciences. Retrieved from https://www.researchgate.net/publi 
cation/277240833._Monitoring_of _Groundwater_Recharge_for_Floo d_Management/figures? $\mathrm{lo}=1 \& u$ utm _source=google\&utm_medium =or ganic. 18/01/2020.

Okoye, I.C., Abu, S.J., Obiezue, N.N.R. \& Ofoezie, I.E. (2014). Prevalence and seasonality of parasites of fish in Agulu Lake, Southeast, Nigeria. African Journal of Biotechnology, 13(3): 502- $508 . \quad$ DOI: 10.5897/AJB2013.13384

Omeji, S., Solomon, S.G. \& Obande, R.A. (2010). A Comparative Study of the Common Protozoan Parasites of Heterobranchuslongifilis from the Wild and Cultured Environments in Benue State. Pakistan Journal of Nutrition, 9 (9): 865-872. ISSN 1680-5194.

Paperna, I. (1980). Parasite, infection and diseases of fishes in Africa. An update. CFA Technical Paper No. 31. Rome, FAO. FAO

Paperna, I. (1996). Parasites infection and disease of fishes in Africa. CIFA Technical Paper No. 31 Food and Agriculture Organization, Rome. FAO.

Roberts, R.J. (1995). Parasitology of Teleosts. In: Fish Pathology. 2nd Edn. London, UK: Wiley Publishers.

Roberts, R.J. (2001). Parasitology of Teleosts. In: Fish Pathology. (3rd ed. Robert R.J (Ed.). Philadelphia: W.B. Saunders.
Tavares-Dias, M. \& Martins, M. L. (2017). An overall estimation of losses caused by diseases in the Brazilian fish farms. J. Parasitic Diseases: Official Organ Indian Soc. Parasitology, 41(4): 913-918.

Tossavi, N.D., Gbankoto, A., Adité, A., Ibikounlé, M.., Grunau, C. \& Sakiti, G.N. (2014). Metazoan parasite communities of catfishes (Teleostei: Siluridae) in Benin (West Africa). Parasitology Research, 113(11): 3973-3983. DOI: 10.1007/s00436-014-4063-x.

Ugbor, O.N., Odo, G.E.,Nwani, C.D., Ochang, S.N.,Somdare, P.O. \& Agbakwuo, C.A.(2014). Parasitic fauna of two dominant Clariid (Siluriformes) catfishes in a tropical freshwater ecosystem, Nigeria. Nigerian Journal of Fisheries, 11 (1 \& 2): 745-755.

Uruku, M.N. \& Adikwu, I.A. (2017). Seasonal prevalence of Clariids fishes from the Lower Benue River, Nigeria. Nigerian Journal of Fisheries and Aquaculture. 5(2): 11-19. ISSN 2350-1537.

Zarlenga, D.S., Hoberg, E.P., Rosenthal, B., Mattiucci, S. \& Nascetti, G. (2014). Anthropogenics: Human Influence on Global and Genetic Homogenization of Parasite Populations. Faculty Publications from the Harold W. Manter Laboratory of Parasitology. 809. Retrieved from http://digitalcommons.unl.edu/para sitologyfacpubs/809. 